\title{
Porphyrins as Red-Emitting Organic Electroluminescent Material
}

\author{
Raghu Nath Bera \\ Department of Physics, Basirhat College, Basirhat, North 24 Parganas, \\ WB 743412, India
}

\begin{abstract}
Organic light emitting diodes (OLEDs) have attracted significant interest because of their potential applications in flat panel displays and in solid state lighting. For full-color displays, it is important to develop blue, green and red light emitting materials. Development of red-emitting OLEDs is particularly important as good redemission with adequate intensity remains a challenge in this field. There are many red-emitting materials, which have been used in OLEDs as emitting materials. Among them, materials based on phorphyrin compounds are highly attracting because most of the porphyrins emit sharp red band. There are a number of porphyrin compounds with or without any metal atom in the central having various electronic and optical properties. The fabrications of devices with porphyrins are also easy by using popular techniques like thermal evaporation, spin coating or by inkjet printing. As a result they are used widely in red-emitting OLEDs for last twenty years or so. An awful lot progress has been made recently in improving the performance of redemitting as well as other devices. In this article, recent progresses of red-emitting devices based on porphyrins are reviewed.
\end{abstract}

Keywords: porphyrin, red emission, EL, fluorescence, phosphorescence, OLED,

\section{Introduction}

The Porphyrins are a class of macrocyclic compounds, which attracted much attention due to their potential applications in various fields of science and technology. Their interesting physical and chemical properties make them an important class of functional materials. The porphyrin ligand helps us to tune desirable material properties that include strong dipole moments and high polarizabilites (Locos O.B. 2006). These materials also offer very interesting nonlinear optical properties due to the molecular level control of energy transfer, which makes them highly useful in the field of optical communications, data storage, electrooptical signal processing (Locos O.B., 2006) and optical switching (Williams D.E., Rietman J.A., Maier J.M., Tan R., Greytak A.B., Smith M.D., Krause J.A. and Shustova B., 2014). Their broad absorption band in UV-Vis region makes them highly useful in preparing light harvesting devices. Porphyrnis are promising sensitizers for wide band gap semiconductors. Dye-sensitized solar cell with high efficiency has been developed through the molecular engineering of porphyrin sensitizers (Mathew S., Yella A., Gao P., Humphry-Baker R., Curchod B.F.E., Ashari-Astani N.,Tavernelli I., Rothlisberger U., Nazeeruddin M. K. and Grätzel M., 2014).

In addition to their technological application, porphyrins, which are widely available in biological compounds have crucial role in the metabolic mechanisms of living bodies. For instance, ironcontaining porphyrins, heme found in blood and the magnesium-containing reduced porphyrin (or chlorine) found in chlorophyll play very important role in living bodies. Porphyrin based biosensor has also been developed for detecting $\mathrm{H}_{2} \mathrm{O}_{2}$ and glucose (Liu Q, Yang Y, Li H, Zhu R, Shao Q, Yang S and $\mathrm{Xu}$ J, 2015). Moreover, photothermal nanodots based on self-assembly of peptide-porphyrin conjugates are used in antitumor therapy (Zou Q., Abbas M.,Zhao L., Li S., Shen G. and Xuehai, 2017).

One of the most exciting applications of porphyrin compounds is in the field of organic light emitting diodes (OLEDs). Recently, OLEDs have showed potential applications in energy efficient flat panel displays and in solid state lighting. For development of full-color flat panel displays we need good blue, green and red emitting materials. These blue, green and red emitting materials are also useful to prepare OLED based low power high luminance light sources. However, the emission of pure red band with sufficient intensity still remains a challenge in the field of OLED. In this context, porphyrin compounds are particularly important as they emit good sharp red band. Their good emission color, decent electrical conductivity along with high 
stability makes them potential candidate in this field. In this article, recent progresses of red-emitting devices based on porphyrins are reviewed. A brief discussion of photophysical properties of porphyrin and OLED have also been done.

\section{Basic Structure of Porphyrin}

The word porphyrin originated from the Greek word 'porphura' meaning purple. They are a large class of deeply colored pigment, of natural or synthetic origin (Giovannetti R., 2012). The basic structural characteristic of porphyrins is a tetrapyrrole macrocyclic ring that consists of $18-\pi$ electron system. The mother compound of this class, which is known as porphyrin, consists of four pyrrole rings that are connected by four methine bridges. The molecular structure of the mother compound is shown in Fig. 1. The positions 2, 3, 7, 8, 12, 13, 17 and 18 are known as $\beta$ - positions and positions 5, 10, 15 and 20 are known as meso-positions (Gouterman M., 1961). The two central pyrrolenine $\mathrm{N}$ atoms of a porphyrin molecule have the ability to accept two protons to form a dication, while two NH groups of the molecule have the ability to donate two protons to form a dianion. The porphyrin dianion has the ability to form metalloporphyrin which contain at least one bond between one of the central $\mathrm{N}$ atoms and a metal ion. This helps porphyrin molecule to bond with metals to create various new compounds having different physical and chemical properties.

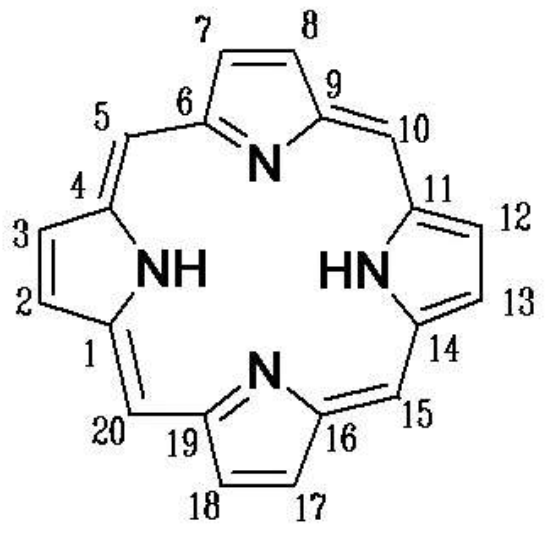

Fig. 1 Molecular structure of porhyrin

\section{Photophysical properties of porphyrins}

Porphyrins are well-known for their intense colors arise from the extensive macrocyclic conjugation of $\pi$-electron systems. The characteristic absorption spectrum of porphyrin consists of two distinct bands, one in the near UV region and the other in the visible region (Weiss, C., Kobayashi H. and Gutterman M., 1965). The absorption in the UV region, which is extremely intense, is known as Sore or B-band and a number of absorptions in the visible region, which are weaker, are known as the Q-band. The intense B- band has a typical extinction co-efficient of the order of $10^{5} \mathrm{M}^{-1} \mathrm{~cm}^{-1}$ and the extinction co-efficient of the Q-band is $10^{4} \mathrm{M}^{-1} \mathrm{~cm}^{-1}$ (Giovannetti R., 2012). UVVis absorption spectrum of a porphyrin strongly depends on the conjugation pathway and symmetry of the molecule (Gouterman M., 1961; Locos O.B., 2006). The metal atom in the center of the molecule has also strong effect on the absorption properties of the porphyrin as it affects the whole array in the ligand part of the molecules. The origin of absorption spectrum of a porphyrin has been explained by fourorbital (two highest occupied $\pi$ orbitals and two lowest unoccupied $\pi *$ orbitals) theorem. There are two highest occupied molecular orbitals (HOMOs) and two lowest unoccupied molecular orbitals (LUMOs) in a porphyrin system and the absorption bands are arises due to the transition between these two HOMO and LUMO levels. The B band arises due to the transition from the ground state to the second excited state (S0 -S2) and Q band arises due to the transition from the ground state to the first excited state (S0-S1).

The fluorescence spectrum of a porphyrin usually consists of two peaks, the emission from the first excited singlet state, $\mathrm{Q}(0,0)$ and a vibronic overtone $\mathrm{Q}(0,1)$ (Locos O.B, 2006). Like absorption, luminescence properties of porphyrin also strongly depend on the central metal atom.

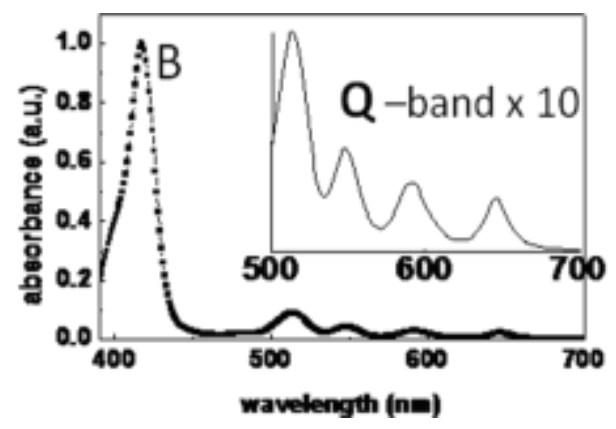

Fig.2 Absorption spectrum of tetraphenyl porphyrin. Inset shows the enlarged Q-band

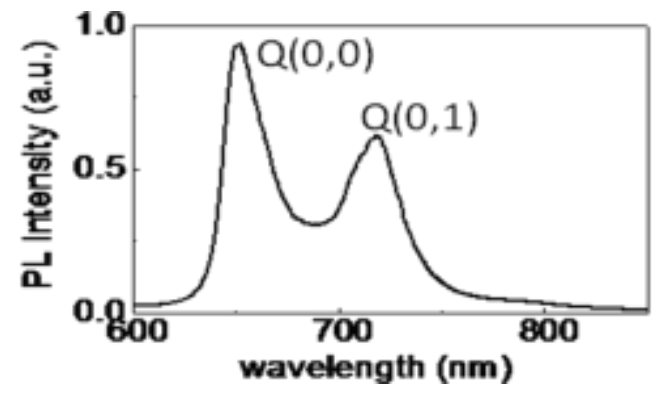

Fig.3 Emission Spectrum of tetraphenyl porphyrin 
For metalloporphyrins, the phosphorescence and fluorescence generally occur at shorter wavelength region than their freebase analogous as a consequence of possessing higher singlet and triplet excitation energy. In addition, a decrease in fluorescence and an increase in phosphorescence intensity have been noticed in the case of metalloporphyrins due to the strong spin-orbit coupling in the presence of heavy atom (Gutterman M.,1961).

\section{Organic Light Emitting Diodes}

Electroluminescence (EL) is the phenomenon of the emission of light from condensed matter under the application of an external electric field. EL from organic material was first reported by Pope et al (Pope M, Kallman H. P. \& Mgnante P. J.,1963). They observed EL from single crystal of anthracene at a bias of about $400 \mathrm{~V}$. However, OLED actually came to the lime light in 1987 when Tang and VanSlyke of Eastman Kodak co, USA, published a paper describing a device consisting of two thin layers of small molecular weight organic materials with diode like structure (Tang C. W. and VanSlyke S. A., 1987). Shortly afterwards, in 1990 Cambridge group led by Sir Richard H. Friend also announced EL from polymer materials (Burroughes J.H., Bradley D.D.C., Brown A.R., Marks R.N., Mackay K, Friend R.H., Burns P.L. and Holmes A.B., 1990).

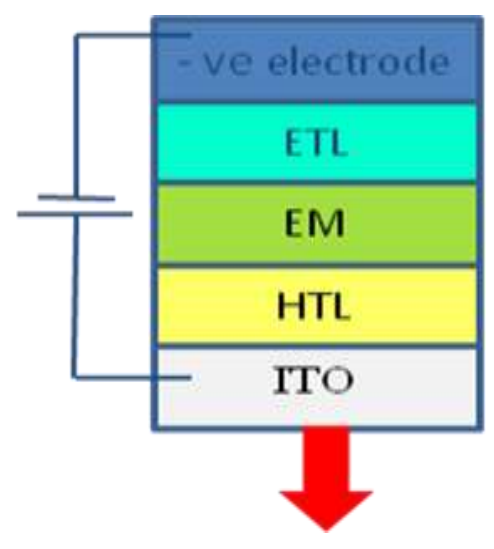

Fig.4 A typical three layer device structure

The LED is a semiconductor device having a $p-n$ junction, which can emit light under forward bias condition. An OLED is a light emitting diode made with organic semiconductors. OLEDs are constructed by sandwiching one or more organic layer/layers in between two suitable electrodes, one of which must be transparent in nature to get the emission from the diode. At the negative electrode (cathode), electrons are injected into the material and at the positive electrode (anode), holes are injected into the material. The two charges move toward each other through the organic layer under the applied electrical field. During their movement if they meet on the same molecular site, they can form a bound electron-hole pair known as exciton, which may decay to give out light. To have an efficient OLED, it is necessary to have balanced charge injection (equal numbers of holes and electrons) and transport, capture of all the injected charges to form excitons, and radiative decay of all the excitons. The simplest OLED has the emissive organic layer sandwiched between two electrodes (Cumpstey N, Bera R.N., Burn P.L. and Samuel I.D.W., 2005). In a single layer device, it is very difficult to achieve a balance between electrons and holes inside the emissive layer, resulting in a less emissive device (Bera R.N., Sakakibara Y., Tokumoto M. and Saito K, 2002). In order to get a balance between electrons and holes inside the emissive layer, multilayer devices were prepared by choosing proper combination of hole injection layer (HIL), hole transporting layer (HTL), electron injecting layer (EIL) and electron transporting layer (ETL) as well as using hole blocking layer (HBL) and electron blocking layer (EBL). In a bilayer or multilayer devices, it is easy to get a balance between electrons and holes inside the emissive layer, resulting in an efficient device (Bera R.N., Cumpstey N., Burn P.L. and Samuel I. D.W.,2007; Lo S.-C., Bera R. N., Harding R. E, Burn P. L. and Samuel I. D. W., 2008). The emission color of a multilayer device depends on the nature of the emissive material.

\section{Porhyrins as Red-Emitting Material in OLED}

5.1 EL properties of porphyrins

Since the report of OLED by Tang, there has been increasing research activities in this field and enormous progress has already been made in the applications of OLED technologies. Different materials were used to prepare OLEDs of different colors. Among different color emitting materials, porphyrins were well-known for their good red emission and a lot of porphyrin family compounds with various molecular structures and electronic states were also available (Fig. 5). So, researcher in this field started to search porphyrin family compounds for new red-emitting materials. As a result there are a number of publications in OLEDs using porphyrin as red-emitting materials.

Porphyrins, in general emit good red band in dilute solution (Fig. 3). However, most of the porphyrins do not emit or emit very low at solid state or even in concentrated solution. The decrease in emission efficiency with the increase in concentration of guest is known as concentration quenching (Bera R.N., Sakakibara Y., Abe S., Yase K. and Tokumoto M., 2005) and often occurs in OLEDs. To overcome this problem porphyrin materials are doped in suitable host material to prepare OLEDs. In this host-guest system, porphyrin guests are excited by the Förster 
type energy transfer from host to the guest (Bera R.N., Sakakibara Y., Tokumoto M. and Yase K., 2003). According to the Förster theory of dipoledipole energy transfer, the energy transfer rate is proportional to the overlap integral between the host emission and guest absorption as shown in Fig.6 (Galanin M. D.,1996). Larger the overlap integral, higher is the energy transfer rate and higher is the efficiency of the device. So to get efficient devices, not only efficient emissive material but also suitable host material is required.
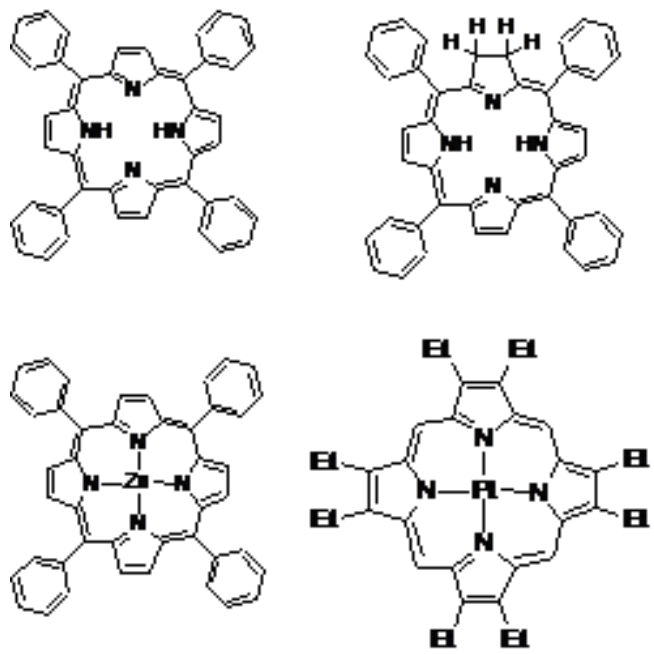

Fig. 5 : Few light emitting porphyrin molecules: (A) top left- TPP, (B) top right-TPC,(C) bottom left $\mathrm{ZnTPP}$ and (D) bottom right-PtOEP

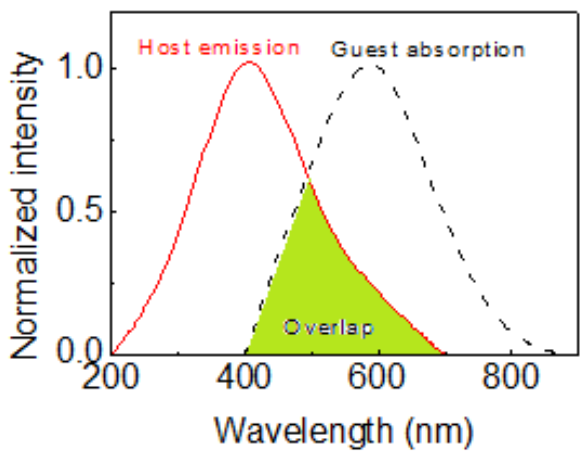

Fig. 6. Schematic diagram of spectral overlap integral between host emission and guest absorption

\subsection{OLEDs based on porphyrins}

A porphyrin compound tetraphenylporphine (TPP) in which phenyl rings are attached in meso- positions 5 , 10, 15 and 20, (as shown in Fig. 5A) is used widely in OLEDs as red-emitting materials. This porphyrin compound emits a good sharp red band with $13 \%$ photoluminescence quantum yield in dilute solution. Burrows et al reported a device in which TPP was doped in a well-known host for red dopant, tris(8hydroxyquinoline) aluminum (Alq3) (Burrows P E, Forrest S R, Thompson ME and Sibley SP, 1996). In this device, green emitting Alq3 acts as ETL and $N$, $N^{\prime} \quad$-diphenyl- $N, \quad \mathrm{~N}^{\prime} \quad$-bis(3-methylphenyl)-1,1'biphenyl-4,4-diamine (TPD) acts as HTL as well as EBL. They observed saturated red emission with CIE co-ordinates $(0.710,0.285)$ from a device with structure ITO/TPD/Alq3+TPP/MgAg. In this device, capture of electron and hole took place near the interface of TPD and Alq3 to form exciton. These excitons then migrated to Alq3 layer, excitation energy transferred to the dopant TPP and finally the emission takes place from TPP sites, resulting in red emission. One interesting observation from their experiment is that they observed saturated red color even at very low doping concentration and they explained it by efficient energy transfer due to the larger Förster radius for TPP in Alq3 (3.3 nm).

Ohmori et al reported TPP: Alq3 OLED in which they improved the performance of the device by using another red emitting dopant 4-(dicyanomethylene)-2-methyl-6- (p-dimethylaminostyryl)-4H-pyran(DCM) along with TPP (Ohmori Y. , Kajii H, Sawatani T. , Ueta H. , Yoshino K, 2001). The structure of their OLED is very similar to that of reported by Burrows et al like ITO/TPD/Alq3+DCM+TPP/Mg-Ag. A two fold increase of efficiency from $0.8 \mathrm{~mW} / \mathrm{A}$ without DCM to $1.6 \mathrm{~mW} / \mathrm{A}$ along with lower driving voltage has been observed when sufficient amount of DCM was co-doped with TPP $(56: 1 \mathrm{~mol} \%)$ in Alq3. Another interesting observation from their experiment is that at lower concentration of DCM the efficiency of the diode was found to decreased with increase of DCM concentration and at a concentration higher than 8:1 mole \% (DCM :TPP), efficiency increased with the increase of DCM concentration. In those co-doped devices, excitation energy was transferred in a twostep process from Alq3 to TPP via DCM. However, they explained that energy transfer from DCM to TPP in their fabricated diode did not take place due to the Förster type energy transfer as the position of DCM emission (430nm) and position of TPP absorption (570-630nm) is not suitable for this type dipole-dipole energy transfer. There might be some other kind of energy transfer occurs in those codoped devices.

OLED based on red emitting TPP have also been reported using zinc complex as host instead of widely used Alq3 (Janghouria M, Mohajerani E, Amini M M and Najafi E, 2014). In a multilayer device with structure, ITO/PEDOT:PSS/PVK/ Zncomplex: TPP/Al, a good red emission with CIE coordinates $(0.70,0.28)$ has been noticed for a doping concentration of $2 \%$ of TPP.

\subsection{OLEDs based on phosphorescence porphyrins}


Another interesting porphyrin compound for OLED application is tetraphenyl chlorin (TPC) (Fig. 5B). Chlorins belong to a reduced porphyrin family in which one or more exo pyrrole double bond is reduced by hydrogen. In ordinary porphyrins, oscillator strength of the Q-band transition is small. So, absorption of $\mathrm{Q}$-band is weak, and hence fluorescence quantum yield is also low. Due to the reduction, oscillator strength of the Q-band transition in chlorin increased and hence the intensity of the corresponding 0-0 transition increased drastically resulted in a two fold increase of PLQY of TPC in dilute solution (0.26) than that of TPP $(0.13$ in dilute solution) (Kalyanasundaram K, 1992). Due to the significant increased of PLQY, TPC became a potential red-emitting candidate in the field of OLEDs and consequently used widely in OLEDs. In the reported device (Sakakibara Y, Okutsu S., Enokida T., Tani T.,1999), OLEDs were prepared by doping TPC in Alq3. They also observed a good red band from their diodes. Like the TPP device, in this case also Förster type energy transfer from Alq3 (host), excited the TPC (guest) molecules. As discussed earlier, the rate of energy transfer depends on the overlap integral of host emission and guest absorption. Alq3 has an intense emission at $550 \mathrm{~nm}$, Q-band absorption of TPC around this region is weak but has a strong B-band from $375 \mathrm{~nm}$ to $475 \mathrm{~nm}$ with peak at 425.The well-known hole-transporting material $N, N^{\prime}$-diphenyl- $N, N^{\prime}$-bis(3-methylphenyl)$1,1^{\prime}$-biphenyl-4,4' -diamine (TPD) has a strong emission band from $375 \mathrm{~nm}$ to $500 \mathrm{~nm}$ with a peak at around $410 \mathrm{~nm}$. Therefore, spectral overlap integral between the TPD emission and the TPC absorption is larger than the overlap integral between Alq3 emission and TPC absorption. So it was expected that energy transfer from TPD to TPC is more efficient than that of between Alq3 to TPC. Indeed, efficient diodes have been observed when TPC was doped in TPD with a suitable HBL (Bera R.N., Sakakibara Y., Tokumoto M. and Saito K, 2002). Device performance was improved further by introducing a proper configuration of 3-(4-biphenyl)4-phenyl-5-(4-tert-butylphenyl)-1,2,4-triazole(TAZ) and Alq3 (ETL) as electron mobility in Alq3 is higher than in TAZ.

Another class of porphyrins is metalloporphyrins. The spectral feature of metalloporphyrin ( both position and intensity) strongly depends on the nature of the central atom. Among various metalloporphyrins, zinc-tetraphenylporphyrin (ZnTPP) (Hamada Y., 1997) and 2,3,7,8,12,13,17,18-octaethyl-21H,23H-porphine platinum(II) (PtOEP) (Baldo M.A., O’Brien D.F., You Y., Shoustikov A., Sibley S., Thompson M.E. and Forrest S.R., 1998) have showed promises in the development of OLEDs. Tetrakis (dihydrxyphenyl) Zinc(II) porphyrin (Zn-TDHPP) is another metalloporphyrin, which has been used as red- emitting material in OLED (Janghouria $M$ and Adinehb M, 2017). With a multilayer device with structure ITO/PEDOT:PSS /PVK:PBD:Zn-TDHPP/ $\mathrm{Al}$ emits a red band from $602 \mathrm{~nm}-645 \mathrm{~nm}$. A change in emission spectrum has also been noticed upon the modification of Zn-TDHPP with different functional group.

Graphene oxide-cobalt porphyrin (GO-CoTPP) hybrid material has also been used as emissive material to prepare red OLED (Janghouria M, 2017). In a multilayer device with structure ITO/ PEDOT:PS /PVK:PBD:GO-CoTPP/ TPBI /Al. A red EL from thin film of PVK: PBD:GO-CoTPP was noticed. However, when Co-TPP was covalently grafted on grapheme oxide sheets, near-white EL was noticed.

Among different metalloporphyrin, PtOEP is an outstanding candidate as this is a phosphorescent material. Excitons generated electrically are highly sensitive to spin, and hence, when they generated, there is a certain probability that they form a singlet or triplet exciton. The ratio of singlets to triplets is $1: 3$ in the case of small molecules. If the emissive material is fluorescent, then only the singlets can emit light, while if it is phosphorescent, both the singlets and triplets can be captured for light emission. Therefore, phosphorescent materials inherently have the potential to form the most efficient devices. Baldo et al reported OLED using PtOEP in 1998 (Baldo M A, O’Brien D F, You Y, Shoustikov A, Sibley S, Thompson M E and Forrest S R, 1998). This was the first reported phosphorescent OLED, which established the utility phosphorescent material to prepare highly efficient OLED. They also used Alq3 as host to prepare their devices because PtOEP has a strong absorption around $530 \mathrm{~nm}$, which is close to the peak emission of Alq3. They found a saturated red emission from their device with multilayer structure (ITO /CuPc / NPD/Alq3+PtEOP/Alq3/MgAg). The peak external and internal quantum efficiency of their device was $4 \%$ and $23 \%$, respectively. They estimated that more than $90 \%$ of the singlet and triplet excitons took part in the emission process. Device efficiency was improved further (O'Brien D F, Baldo $\mathrm{M} \mathrm{A}$, Thompson M E and Forrest S R, 1999) by using another host material, 4,4'-N,N'-dicarbazolebiphenyl (CBP). The peak external quantum efficiency of $5.6 \%$ was achieved with a device configuration of ITO/NPD/CBP+PtOEP/BCP/Alq3/MgAg. In this system, weak spectral overlap between PtOEP absorption and CBP emission, reduced nonradiative recombination. However, energy transfer from CBP to PtOEP is also weak. In compensation, a thin layer of 2,9-dimethyl-4,7 diphenyl-1,10-phenanthroline (bathocuproine, or $\mathrm{BCP}$ ) was used as a barrier to exciton diffusion in CBP, improving the energy transfer to PtOEP. In addition, a capping layer of 
Alq3 (20nm) was used to prevent quenching of PtOEP excitons at the negative electrode.

Near IR emissive OLEDs with color ranging from $771 \mathrm{~nm}-1005 \mathrm{~nm}$ has been noticed from few other platinum(II) porphyrin complexes (Graham K.R., Yang Y., Sommer J.R., Shelton A.H., Schanze K.S., Xue J. and Reynolds J.R., 2011). But detail discussions about these IR OLEDs are beyond the scope of this review.

\section{Conclusions}

The biochemical and optical properties of porphyrins have been a matter of continuing scientific interest for decades. More recently, they have emerged as an attractive class of optelectronic material for preparing red-emitting OLEDs due to their sharp red emission. The physical properties of a porphyrin strongly depend on the conjugation pathway and symmetry of the molecule. The metal atom in the center of the molecule has also strong effect on the physical properties of the porphyrin as it affects the whole array in the ligand part of the molecules. Therefore, one can design a porphyrin molecule to his/her own choice for suitable application. Nowadays, though porphyrins are used substantially in OLEDs, but their low emission efficiency in pure solid form is a limitation of this materials. To overcome this problem OLEDs based on porphyrin materials are prepared by doping these materials in suitable host materials like Alq3, TPD, CBP etc. The choice of the right porphyrin molecules along with suitable host offers the highly efficient system for fabricating OLEDs. One of the most exciting properties of few metalloporphyrins like PtOEP is the EL from both singlet and triplet state, which helps us to prepare devices with internal quantum efficiency $100 \%$ with suitable combination of host and guest.

\section{References}

[1] Baldo M.A., O’Brien D.F., You Y., Shoustikov A., Sibley S., Thompson M.E. and Forrest S.R., Highly efficient phosphorescent emission from organic electroluminescent devices, Nature, vol 395(10), pp.151-154 (1998).

[2] Bera R.N., Cumpstey N., Burn P.L. and Samuel I. D.W., Highly branched phosphorescent dendrimers for efficient solution-processed organic light-emitting diodes, Advanced Functional Materials, vol 17, pp.1149-1152 (2007).

[3] Bera R.N., Sakakibara Y., Tokumoto M and Saito K, Enhancement of red electroluminescence from device with tetraphenylchlorin doped into a hole-transporting material by improving electron transporting property, Japanese Journal of Applied Physics Part 2 Letter, vol 41, pp.L1010-L1012 (2002).;
ISSN 2455-6378

Red-emitting organic electroluminescent devices with tetraphenylchlorin doped into a holetransporting material, Japanese Journal of Applied Physics Part 2 Letter, vol 41, pp.L391L393 (2002).

[4] Bera R.N., Sakakibara Y., Tokumoto M. and Yase K., Time-resolved photoluminescence study on energy transfer from Alq3 (tris(8hydroxyquinoline)aluminum) to red-emissive ttraphenylchlorin, Japanese Journal of Applied Physics Part 1, vol 42(12), pp.7379-7380, (2003).

[5] Bera R.N., Sakakibara Y., Abe S., Yase K. and Tokumoto M., Time-resolved photoluminescence study on concentration quenching of a red emitting tetraphenylchlorin dye for organic electroluminescent devices, Synthetic Metals, vol 150, pp.9-13 (2005).

[6] Bera R.N., Sakakibara Y. and Tokumoto M., Concentration quenching of a red emitting electroluminescent dye tetraphenylporphyrin: A time-resolved photoluminescence study, Journal of Materials Science-Materials in Electronics, vol 16, pp.549-552 (2005).

[7] Burroughes J.H., Bradley D.D.C., Brown A.R., Marks R.N., Mackay K, Friend R.H., Burns P.L. and Holmes A.B., Light emitting diodes based on conjugated polymers, Nature, vol 34, pp.539541 (1990).

[8] Burrows P.E., Forrest S.R., Thompson M.E. and Sibley S.P., Color-tunable organic-light emitting devices, Applied Physics Letters, vol 69, pp.2959-2961 (1996).

[9] Cumpstey N, Bera R.N., Burn P.L. and Samuel I.D.W., Investigating the effect of steric crowding in phosphorescent dendrimers, Macromolecules, vol 38, pp.9564-9570 (2005).

[10]Galanin M. D., Luminescence of Molecules and Crystals, Cambridge International Science Publishing pp.66-70 (1996).

[11]Giovannetti R. The Use of Spectrophotometry UV-Vis for the Study of Porphyrins, Macro To Nano Spectroscopy, Dr. Jamal Uddin (Ed.), ISBN: 978-953-51-0664-7, InTech, pp.1-8 (2012), Available from:http://www.intechopen.com/books/macroto-nano-spectroscopy/the-use-ofspectrophotometry-uv-vis-for-thestudy-ofporphyrins

[12]Graham K.R., Yang Y., Sommer J.R., Shelton A.H., Schanze K.S., Xue J. and Reynolds J.R., Extended conjugation platinum(II) porphyrin for use in near-infrared emitting organic light emitting diodes, Chemistry of Materials, vol 23, pp.5305-5312 (2011).

[13]Goutterman M, Spectra of porphyrin, Journal of Molecular Spectroscopy, vol 6, pp.138-163 (1961).

[14]Hamada Y, The development of chelate metal complex as an organic electroluminescent 
material, IEEE Transaction of Electronic Devices, vol 44, pp.1208-1217 (1997).

[15]Janghouria M, Mohajerani E, Amini M M and Najafi E, Red organic emitting device based on TPP and a new host material, Applied Physics A, vol 114(2), pp.445-451 (2014).

[16]Janghouria $M$ and Adinehb $M$, Color optimization of red organic light emitting diodes (OLEDs) through dihydroxyphenyl -substituted zinc porphyrin emitters, Journal of Photochemistry and Photobiology A: Chemistry, vol 341, pp.31-38 (2017).

[17]Janghouria M, Electroluminescence and photoluminescence from a fluorescent cobalt porphyrin grafted on graphene oxide, Journal of Electronic Materials, vol 46(10), pp.5635-5641 (2017).

[18]Kalyanasundaram K, Photochemisyry of Polypyridine and Porphyrin Complex, Academic Press, London, (1992).

[19]Liu Q, Yang Y, Li H, Zhu R, Shao Q, Yang S and $\mathrm{Xu} \mathrm{J}, \mathrm{NiO}$ nanoparticles modified with 5,10,15,20-tetrakis(4-carboxylpheyl)-porphyrin: promising peroxidase mimetics for $\mathrm{H} 2 \mathrm{O} 2$ and glucose detection, Biosensors and Bioelectronics, vol 64, pp.147-153 (2015).

[20]Lo S.-C., Bera R. N., Harding R. E, Burn P. L. and Samuel I. D. W., Solution-processible phosphorescent blue dendrimers based on biphenyl-dendrons and factris(phenyltriazolyl)iridium(III) cores, Advanced Functional Materials, vol 18, pp.3080-3090 (2008).

[21]Locos O.B. Synthesis and investigations of novel alkenylporphyrins and bis(porphyrins), The Queensland university of technology, pp.115 (2006).

[22]Mathew S., Yella A., Gao P., Humphry-Baker R., Curchod B.F.E., Ashari-Astani N.,Tavernelli I., Rothlisberger U., Nazeeruddin M. K. and Grätzel M., Dye-sensitized solar cells with $13 \%$ efficiency achieved through the molecular
ISSN 2455-6378

engineering of porphyrin sesitizers, Nature Chemistry, vol 6, pp.242-247 (2014).

[23]O’Brien D.F., Baldo M.A., Thompson M.E. and Forrest S.R., Improved energy transfer in electrophosphorescent devices, Applied Physics Letters, vol 74(3), pp.442-444 (1999).

[24]Ohmori Y., Kajii H, Sawatani T. , Ueta H. and Yoshino K., Enhancement of electroluminescence utilizing confined energy transfer for red light emission, Thin Solid Films, vol 393, pp.407-411 (2001).

[25]Pope M, Kallman H. P. and Mgnante P, Electroluminescence in organic crystal, Journal of Chemical Physics, vol 38, pp. 2042-2043 (1963).

[26]Sakakibara Y, Okutsu S., Enokida T., Tani T., Red organic electroluminescence devices with a reduced porphyrin compound, tetraphenylchlorin, Applied Physics Letters, vol 74, pp.2587-2589 (1999).

[27]Tang C. W and VanSlyke S. A, Organic electroluminescent diodes, Applied Physics Letters, vol 51, pp.913-915 (1987).

[28]Weiss, C., Kobayashi H. and Gutterman M., Spectra of porphyrins, Part III1 Self-consistant molecular orbital calculation of porphyrin and related ring system, Journal of Molecular Spectroscopy, vol 16, 415-450 (1965).

[29]Williams D.E., Rietman J.A., Maier J.M., Tan R., GreytakA.B., Smith M.D., KrauseJ.A. and Shustova B., Energy transfer on demand: Photoswitch-Directed behavior of metalporhyrin frameworks, Journal of the American Chemical Society, vol 136, pp.11886-11889 (2014).

[30]Zou Q., Abbas M.,Zhao L., Li S., Shen G. and Xuehai, Biological photothermal nanodots based on self-assembly of peptide-porphyrin conjugates for antitumor therapy, Journal of the American Chemical Society, vol 139, pp.19211927 (2017). 